effects. In the first, it was used as an adjunct therapy with zidovudine for inhibiting viral replication in HIV-infected patients (16) at a dose of $3 \mathrm{~g} / \mathrm{d}$ (equivalent to $43 \mathrm{mg} / \mathrm{kg} / \mathrm{d}$ in a $70-\mathrm{kg}$ person); in the other, it was used as substrate reduction therapy for Gaucher's type I disease at $300 \mathrm{mg} / \mathrm{d}$ (equivalent to $4.3 \mathrm{mg} / \mathrm{kg} / \mathrm{d}$; ref. 17) as described above. The contraceptive effect of the drug observed in mice seems to occur at a dose of $5 \mathrm{mg} / \mathrm{kg} / \mathrm{d}$, therefore setting a benchmark for future clinical studies of the effects of this drug on contraception in humans. However, as the authors point out, Gba2 is more highly expressed in mouse testes than GBA2 is in human testes. Whether or not NB-DNJ has a similar contraceptive effect on humans therefore remains an open question.

In conclusion, results from the Gba2 knockout model (7) provide an interesting insight into glycolipid metabolism in the testis. In the future, this model may prove to be a valuable resource in the investigation of the male contraceptive effects of NB-DNJ, one of the prime candidates for the ever-elusive nonhormonal male contraceptive pill.

\section{Acknowledgments}

The authors thank Richard N. Sifers (Baylor College of Medicine) for his insight- ful comments and critical review of this commentary.

Address correspondence to: Martin M. Matzuk, Department of Pathology, One Baylor Plaza, Baylor College of Medicine, Houston, Texas 77030, USA. Phone: (713) 798-6451; Fax: (713) 798-5833; E-mail: mmatzuk@bcm.tmc.edu.

Angshumoy Roy, Yi-Nan Lin, and Martin M. Matzuk contributed equally to this work.

1. Kolter, T., and Sandhoff, K. 2006. Sphingolipid metabolism diseases. Biochim. Biophys. Acta. In press.

2. Fujimoto, H., et al. 2000. Requirement of seminolipid in spermatogenesis revealed by UDP-galactose: ceramide galactosyltransferase-deficient mice. J. Biol. Chem. 275:22623-22626.

3. Takamiya, K., et al. 1998. Complex gangliosides are essential in spermatogenesis of mice: possible roles in the transport of testosterone. Proc. Natl. Acad. Sci. U. S. A. 95:12147-12152.

4. Butler, A., et al. 2002. Reproductive pathology and sperm physiology in acid sphingomyelinase-deficient mice. Am. J. Pathol. 161:1061-1075.

5. Tybulewicz, V.L., et al. 1992. Animal model of Gaucher's disease from targeted disruption of the mouse glucocerebrosidase gene. Nature. 357:407-410.

6. Cox, T.M., et al. 2003. The role of the iminosugar $\mathrm{N}$-butyldeoxynojirimycin (miglustat) in the management of type I (non-neuronopathic) Gaucher disease: a position statement. J. Inherit. Metab. Dis. 26:513-526.

7. Yildiz, Y., et al. 2006. Mutation of $\beta$-glucosidase 2 causes glycolipid storage disease and impaired male fertility. J. Clin. Invest. 116:2985-2994. doi:10.1172/ JCI29224.

8. Van der Spoel, A.C., et al. 2002. Reversible infertility in male mice after oral administration of alkylated imino sugars: a nonhormonal approach to male contraception. Proc. Natl. Acad. Sci. U. S. A. 99:17173-17178.

9. Matern, H., Heinemann, H., Legler, G., and Matern, S. 1997. Purification and characterization of a microsomal bile acid beta-glucosidase from human liver. J. Biol. Chem. 272:11261-11267.

10. Matzuk, M.M., and Lamb, D.J. 2002. Genetic dissection of mammalian fertility pathways. Nat. Med. 8(Suppl.):s33-s40.

11. Kang-Decker, N., Mantchev, G.T., Juneja, S.C., McNiven, M.A., and van Deursen, J.M. 2001. Lack of acrosome formation in Hrb-deficient mice. Science. 294:1531-1533.

12. Yao, R., et al. 2002. Lack of acrosome formation in mice lacking a Golgi protein, GOPC. Proc. Natl. Acad. Sci.U. S. A. 99:11211-11216.

13. Ikonen, E. 2001. Roles of lipid rafts in membrane transport. Curr. Opin. Cell Biol. 13:470-477.

14. Zhang, Y., et al. 2005. Testis-specific sulfoglycolipid, seminolipid, is essential for germ cell function in spermatogenesis. Glycobiology. 15:649-654.

15. Van Weely, S., Brandsma, M., Strijland, A., Tager, J.M., and Aerts, J.M. 1993. Demonstration of the existence of a second, non-lysosomal glucocerebrosidase that is not deficient in Gaucher disease. Biochim. Biophys. Acta. 1181:55-62.

16. Fischl, M.A., et al. 1994. The safety and efficacy of combination N-butyl-deoxynojirimycin (SC48334) and zidovudine in patients with HIV-1 infection and 200-500 CD4 cells/mm3. J. Acquir. Immune Defic. Syndr. 7:139-147.

17. Cox, T., et al. 2000. Novel oral treatment of Gaucher's disease with N-butyldeoxynojirimycin (OGT 918) to decrease substrate biosynthesis. Lancet. 355:1481-1485.

\title{
The paradoxical patent ductus arteriosus
}

\author{
Kathryn N. Ivey and Deepak Srivastava
}

\begin{abstract}
Gladstone Institute of Cardiovascular Disease, Department of Pediatrics, and Department of Biochemistry and Biophysics,
\end{abstract} University of California San Francisco, San Francisco, California, USA.

\begin{abstract}
The ductus arteriosus (DA) is a vessel whose patency is required for fetal survival but is incompatible with postnatal life. Because of developmental insufficiency, the DA in preterm infants often fails to close in a condition known as patent DA (PDA). Although COX inhibitors can be used to close the PDA by lowering circulating prostaglandin levels, their effectiveness is correlated with birth weight, and severely premature infants often require surgical repair. Paradoxically, targeted deletion of $\mathrm{COX}$ pathway components in mice results in PDA. In this issue of the JCI, Yokoyama et al. describe dual roles for prostaglandins in DA development and closure, offering new insights into the mechanism of negative effects of COX inhibitors that may influence the treatment of severely premature infants with PDA and lead to improvement of their outcomes (see the related article beginning on page 3026).
\end{abstract}

Nonstandard abbreviations used: DA, ductus arteriosus; HA, hyaluronic acid; HAS2, HA synthase 2; PDA, patent DA.

Conflict of interest: The authors have declared that no conflict of interest exists.

Citation for this article: J. Clin. Invest. 116:2863-2866 (2006). doi:10.1172/JCI30349.
The ductus arteriosus (DA) is a fetal vessel that bridges the pulmonary and systemic circulation during gestation, when oxygenation of fetal blood occurs in the placenta (Figure 1). In utero, circulating prostaglandins, formed by the action of
COX enzymes, exert their vasodilatory effect on the DA to maintain its patency (1). Activation of the $G$ protein-coupled receptor $\mathrm{EP} 4$ by $\mathrm{PGE}_{2}$, the primary prostaglandin regulating ductal tone, leads to DA smooth muscle relaxation (1). During late gestation, in preparation for birth, SMCs within the DA migrate toward the endothelial lining, forming mounds known as intimal cushions (2). A sharp decline in prostaglandin levels at birth results in constriction of the DA, bringing the intimal cushions into contact and occluding the DA lumen (1).

Failure of DA closure, called patent DA (PDA), is the third most common congenital heart defect, and its incidence is increased in preterm infants (3). Fortunately, pharmacological inhibition of the COXs is an effective means of closing 
Fetal circulation

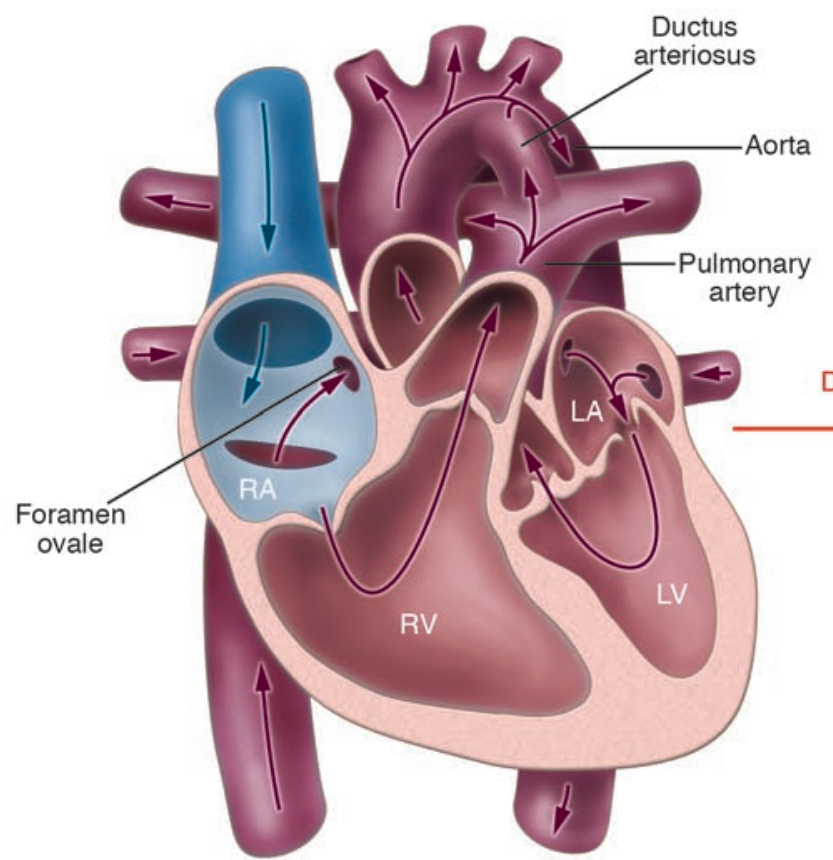

Neonatal circulation

Figure 1

DA during fetal-neonatal circulatory transition. Left: During fetal development, the DA shunts blood away from the lungs and directly into the systemic circulation via the aorta. Right: At birth, as PGE levels fall, SMCs in the DA constrict, bringing the intimal cushions of the DA into contact and occluding the DA lumen, so that blood flows to the neonatal lungs. This remodeling process supports the change from a fetal circulation, where blood is oxygenated in the placenta, to a neonatal circulation, where gas exchange occurs in the lungs. Blue represents oxygen-poor blood; red represents oxygen-rich blood. RA, right atrium; LA, left atrium.

the DA postnatally $(4,5)$. However, COX inhibitors are less effective for DA closure in severely premature infants $(4,5)$, a fact that has long been attributed to the immaturity of their DA smooth muscle and its inability to contract, even as prostaglandins are withdrawn. Conversely, in the case of some congenital heart defects, systemic blood flow relies on maintenance of DA patency, in which case prostaglandins are administered to keep the DA open until surgical repair of the heart or vessels can be performed.

\section{The paradox of PDA}

Given the known role of prostaglandins in maintaining DA patency, a logical corollary would be that mice with targeted deletions of genes that negatively affect prostaglandin synthesis or signaling would result in premature closure of the DA. Surprisingly, mice lacking the DAenriched prostaglandin receptor EP4, or mice with combined deficiency of the COX enzymes, COX-1 and COX-2, were found to have PDA $(6,7)$. How is it that depleting a signaling pathway in mice has the same effect as activating that pathway in humans?

Yokoyama et al. shed light on this question in their study published in this issue of the JCI (8). They establish that $\mathrm{PGE}_{2}$, acting via its receptor, EP4, has an additional prenatal role in the maturation of the fetal DA, preparing it for closure at birth by promoting intimal cushion formation (Figure 2). Using agonists and antagonists of the EP4 receptor, the authors show that EP4 controls DA smooth muscle migration to form intimal cushions while stimulating synthesis and secretion of hyaluronic acid (HA; also known as hyaluronan), an extracellular matrix component through which the SMCs traverse. With elegant use of siRNA-mediated knockdown of HA synthase 2 (HAS2), as well as HA depletion from EP4-activated cultures, the authors confirm that PGE-induced SMC migration occurs through EP4 stimulation of HA production.

These findings offer a likely explanation for why mice lacking prostaglandin signaling in the DA do not experience pre- mature closure of the fetal vessel. EP4-null mice fail to form intimal cushions, so the vasodilatory effects of prostaglandins are likely not required for maintenance of DA patency before birth. Instead, patency persists postnatally in the absence of prostaglandin signaling because, without intimal cushion formation, occlusion of the DA lumen cannot occur.

While we believe that the study by Yokoyama et al. (8) is the first to uncover a role for prostaglandins in intimal cushion formation, the idea that DA remodeling is more important than vasoconstriction for DA closure is not new. The DA of mice lacking smooth muscle myosin, which is required for contraction of DA smooth muscle, also closes appropriately after birth (9), demonstrating that functional closure can occur without vessel wall contraction. Interestingly, comparison of the effects of genetic COX deficiency and administration of COX inhibitors at varying points of mouse gestation also revealed that prostaglandins have a novel role in the development of the DA, beyond their direct vasodilatory effect (10). 


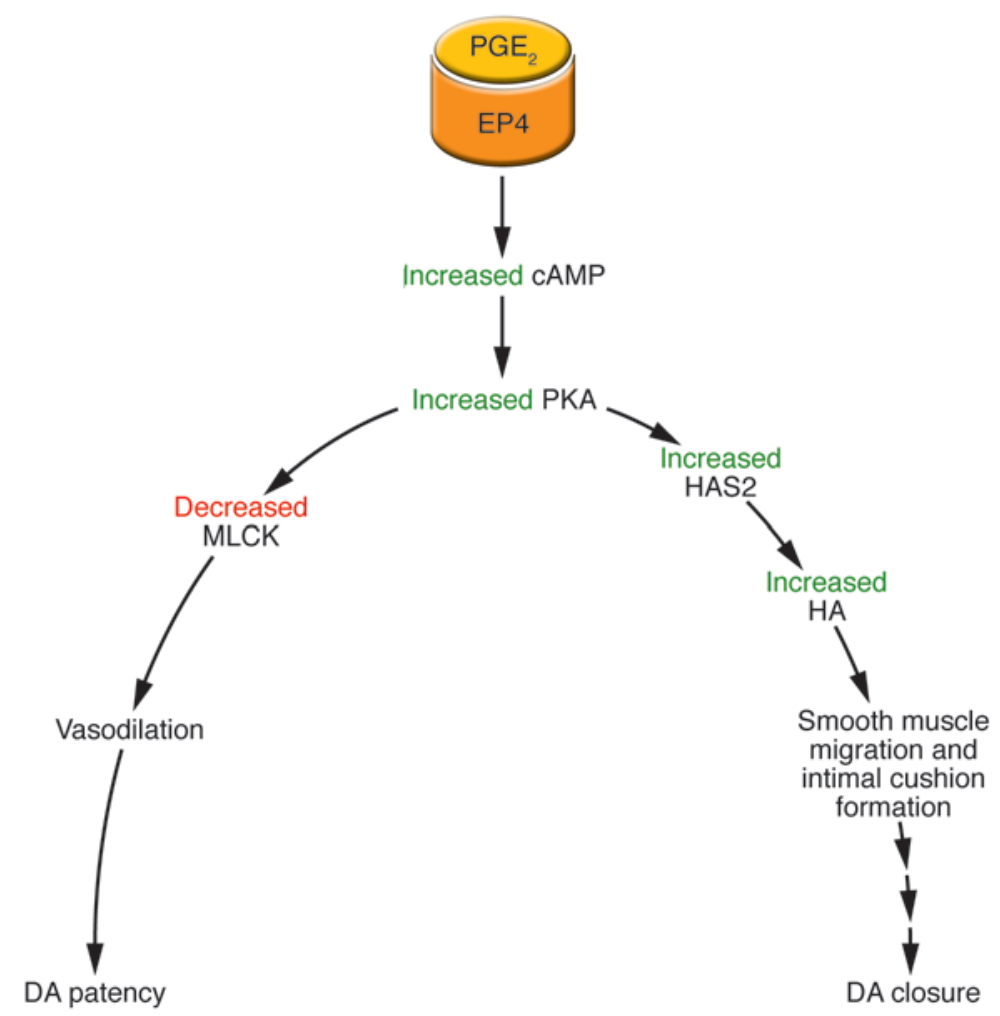

In addition to explaining the paradox of PDA in mice deficient in prostaglandin signaling, the elucidation by Yokoyama et al. (8) of a developmental role for prostaglandins during DA maturation may also add to our understanding of important clinical findings that have yet gone unexplained. In spite of their vasoconstrictive effect on the DA at birth, use of COX inhibitors by pregnant mothers increases the incidence of PDA in newborns $(11,12)$. If prostaglandin signaling is, indeed, required for intimal cushion formation in humans as in rodents, then infants who develop under conditions of insufficient PGE, as in the case of maternal use of COX inhibitors, may be born with an underdeveloped DA that remains patent in spite of PGE withdrawal. The current results also imply that, while DA smooth muscle development is not as advanced in premature infants as in their full-term counterparts, DA smooth muscle immaturity may not be the primary cause for the lack of DA constriction in response to COX inhibitors, as previously thought. Instead, a lack of intimal cushions, whose further development is blocked by the delivery of COX inhibitors, may preclude DA closure in preterm infants.

\section{Implications for pharmacological intervention for PDA}

While COX inhibitors offer an effective means to close the DA in near-term infants, for severely premature babies, COX inhibitors may exacerbate the problem by preventing the formation of intimal cushions required for closure. Yokoyama et al. (8) suggest that HAS activation, combined with prostaglandin blockade, might be an effective means to treat PDA in severely premature infants. Certainly their results suggest that, upon appropriate delivery of directed HAS stimulation, this may be a successful tactic in rodents, and future studies will determine whether a combined therapy is sufficient to promote DA maturation and closure in a timely manner.

It is important to note that, while the underlying molecular pathways controlling DA development and closure are strikingly similar in rodents and larger mammals, there are significant differences in their developmental physiology and the timing of critical closure events. For instance, while functional closure of the DA happens quite rapidly in rodents, occurring within the first few hours after birth (13), normal closure in newborn humans takes place over several days (14). More relevant to the early effects of prostaglandins, inti-

\section{Figure 2}

PGE both positively and negatively affects patency of the DA. Binding of $P E_{2}$ to its receptor, EP4, in the DA results in accumulation of CAMP and activation of PKA. This, in turn, has two somewhat opposing effects on the DA. PKA activation directly promotes vasodilation by inhibiting myosin light chain kinase (MLCK) to maintain DA patency during fetal development. PKA also increases activity of HAS2, leading to HA production that promotes migration of DA SMCs. This process is necessary for formation of intimal cushions that are ultimately required for functional closure of the DA at birth. mal cushion formation occurs much earlier and is far more pronounced in larger mammals than in small rodents (15). It has been hypothesized that, due to their increased luminal diameter, larger mammals require highly developed intimal cushions for contraction of the surrounding smooth muscle to effectively close the lumen (15). So while mice, rats, and even rabbits offer amenable models for genetic and initial pharmacological studies of DA development and closure, other large animals, such as lambs, pigs, or dogs, must be used to more directly model the physiology and pathophysiology of the human DA. Given the impact and potential clinical application of the study by Yokoyama et al., further studies of this kind will no doubt be undertaken in larger model organisms, hopefully offering answers that can be directly applicable to neonatal medicine and improve the outcomes of the multitude of low-birth-weight babies that struggle with this disease.

Address correspondence to: Deepak Srivastava, Gladstone Institute of Cardiovascular Disease, 1650 Owens Street, San Francisco, California 94158,USA. Phone:(415)734-2716; Fax: (415) 355-0141; E-mail: dsrivastava@ gladstone.ucsf.edu. 
1. Smith, G.C. 1998. The pharmacology of the ductus arteriosus. Pharmacol. Rev. 50:35-58.

2. Gittenberger-de Groot, A.C. 1977. Morphology of the normal human ductus arteriosus. In The ductus arteriosus, report of the 75th Ross Conference on Pediatric Research. M.A. Heymann and A.M. Rudolph, editors. Ross Laboratories. Columbus, Ohio, USA. 3-9.

3. Hoffman, J.I., and Kaplan, S. 2002. The incidence of congenital heart disease. J. Am. Coll. Cardiol. 39:1890-1900.

4. Heymann, M.A., Rudolph, A.M., and Silverman, N.H. 1976. Closure of the ductus arteriosus in premature infants by inhibition of prostaglandin synthesis. N. Engl.J. Med. 295:530-533.

5. Friedman, W.F., Hirschklau, M.J., Printz, M.P., Pitlick, P.T., and Kirkpatrick, S.E. 1976. Pharmacologic closure of patent ductus arteriosus in the premature infant. N. Engl. J. Med. 295:526-529.
6. Nguyen, M., et al. 1997. The prostaglandin receptor EP4 triggers remodelling of the cardiovascular system at birth. Nature. 390:78-81.

7. Loftin, C.D., et al. 2001. Failure of ductus arteriosus closure and remodeling in neonatal mice deficient in cyclooxygenase-1 and cyclooxygenase-2. Proc. Natl. Acad. Sci. U. S. A. 98:1059-1064.

8. Yokoyama, U., et al. 2006. Chronic activation of the prostaglandin receptor EP4 promotes hyaluronanmediated neointimal formation in the ductus arteriosus. J. Clin. Invest. 116:3026-3034. doi:10.1172/ JCI28639.

9. Morano, I., et al. 2000. Smooth-muscle contrac tion without smooth-muscle myosin. Nat. Cell Biol. 2:371-375.

10. Reese, J., Anderson, J.D., Brown, N., Roman, C., and Clyman, R.I. 2006. Inhibition of cyclooxygenase isoforms in late- but not mid-gestation decreases contractility of the ductus arteriosus and prevents postnatal closure in mice. Am. J. Physiol. Regul. Integr. Comp. Physiol. doi:10.1152/ajpregu.00259.2006.

11. Norton, M.E., Merrill, J., Cooper, B.A., Kuller, J.A., and Clyman, R.I. 1993. Neonatal complications after the administration of indomethacin for preterm labor. N. Engl. J. Med. 329:1602-1607.

12. Hammerman, C., et al. 1998. Indomethacin tocolysis increases postnatal patent ductus arteriosus severity. Pediatrics. 102:E56.

13. Tada, T., and Kishimoto, H. 1990. Ultrastructural and histological studies on closure of the mouse ductus arteriosus. Acta Anat. (Basel). 139:326-334.

14. Gentile, R., et al. 1981. Pulsed Doppler echocardiographic determination of time of ductal closure in normal newborn infants. J. Pediatr. 98:443-448.

15. Bergwerff, M., DeRuiter, M.C., and Gittenbergerde Groot, A.C. 1999. Comparative anatomy and ontogeny of the ductus arteriosus, a vascular outsider. Anat. Embryol. (Berl.). 200:559-571.

\title{
Role for IKK2 in muscle: waste not, want not
}

\author{
Michael Karin \\ Laboratory of Gene Regulation and Signal Transduction, Department of Pharmacology, School of Medicine, \\ University of California San Diego, La Jolla, California, USA.
}

\begin{abstract}
Activation of transcription factor NF- $\kappa B$, the major regulator of the inflammatory response, depends on the inhibitor of NF- $K B$ kinase (IKK) complex, which is composed of 2 catalytic subunits, IKK1 and IKK2 (also known as IKK $\alpha$ and IKK $\beta$ ), and a regulatory subunit, IKK $\gamma$ (also known as NEMO). In this issue of the JCI, Mourkioti et al. show that muscle-specific disruption in mice of the gene encoding IKK2 prevents NF- $\kappa B$ activation in response to denervation or toxin-induced injury (see the related article beginning on page 2945). Importantly, this genetic manipulation prevents muscle wasting, thereby providing strong evidence in support of a major pathogenic role for inflammation in a variety of muscular dystrophies characterized by progressive muscle fiber degeneration.
\end{abstract}

Transcription factor NF- $\mathrm{KB}$ is one of the major activators of the inflammatory response (1). In most cells, the majority of the NF- $\kappa \mathrm{B}$ pool resides in the cytoplasm but undergoes rapid nuclear translocation upon activation of innate immune receptors or exposure to proinflammatory cytokines, such as TNF- $\alpha$ or IL-1. Nuclear translocation of NF- $\kappa \mathrm{B}$ or its activation depends on degradation of specific inhibitory proteins called inhibitors of NF- $\mathrm{KB}$

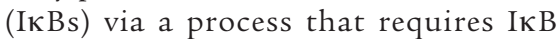
phosphorylation and polyubiquitination (2). Phosphorylation of IкBs is mediated by a specialized protein kinase complex, the IKB kinase (IKK) complex, which is

Nonstandard abbreviations used: I $\mathrm{K} B$, inhibitor of NF-кB; IKK, IкB kinase; MuRF1, muscle ring finger protein 1.

Conflict of interest: The author has declared that no conflict of interest exists.

Citation for this article: J. Clin. Invest. 116:2866-2868 (2006). doi:10.1172/JCI30268. composed of 2 catalytic subunits, IKK1 (also known as IKK $\alpha$ ) and IKK2 (also known as IKK $\beta$ ), and a regulatory subunit called NEMO or IKK $\gamma$ (3). Gene disruption experiments have established that in most cell types, in response to most

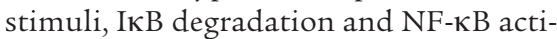
vation are mainly dependent on IKK2 (4). Hence, targeted disruption of the IKK2 gene has been used to demonstrate its involvement in a variety of inflammatory disorders, such as multiorgan dysfunction (5), colitis-associated cancer (6), and even obesity-induced insulin resistance (7). Now, in this issue of the JCI, Mourkioti et al. (8) report on the addition of muscle degeneration and atrophy to the list of pathologies that are remarkably ameliorated upon targeted deletion of IKK2. This suggests that muscle degenerative diseases may have a common inflammatory component and thus may respond to antiinflammatory therapy.

\section{A role for inflammation} in muscular dystrophies?

Muscle degeneration and atrophy is a rather common pathology associated with a variety of diseases, including those that specifically target the muscle, for instance muscular dystrophies (9), or those that target other organs, such as cancer and immune disorders $(10,11)$. Even old age and immobilization can result in muscle wasting. Yet apart from cachexia, in which TNF- $\alpha$ plays an important role, and specific muscle inflammation/myositis, muscular dystrophies in particular and muscle atrophy in general have not been universally thought of as inflammatory diseases. Yet the new work of Mourkioti et al. (8) as well as previous work by Shoelson's group that demonstrated severe muscle wasting following the constitutive activation of NF-KB in muscle cells (12) clearly demonstrate the involvement of the $\mathrm{IKK} / \mathrm{NF}-\mathrm{\kappa B}$ signaling system in different types of muscle degeneration. These data therefore strongly suggest that muscular dystrophies and atrophies should also be considered inflammatory diseases and raise the prospects of novel therapies that target IKK2 or other steps in the NF-кB activation pathway.

Mourkioti et al. (8) used a mouse strain homozygous for a conditional Ikk2 allele (a so-called floxed allele) that can be deleted upon expression of Cre-recombinase to specifically inactivate IKK2 in 